\title{
Prolonged Inhibitory Effects of Repeated Tibial Nerve Stimulation on the Micturition Reflex in Decorticated Rats
}

\author{
Junyan Mai, MD ${ }^{1, a}$; Junhao Liao, MD ${ }^{1, a}$; Yuying Zhang, MD, PhD ${ }^{1, a}$; \\ Baoyi Zhu, MD ${ }^{1}$; Chonghe Jiang, MD $^{1,2}$; Sivert Lindström, MD, PhD $^{2}$; \\ Jianwen Zeng, MD ${ }^{1}$
}

\begin{abstract}
Objective: This study aimed to determine whether a short-term repeated stimulation of tibial nerve afferents induces a prolonged modulation effect on the micturition reflex in a decorticated rat model.

Material and Methods: Fifteen female Sprague-Dawley rats (250-350 g) were fully decorticated and paralyzed in the study. Tibial nerve stimulation (TNS) was delivered by inserting two pairs of needle electrodes close to the nerves at the level of the medial malleolus. Constant flow cystometries $(0.07 \mathrm{~mL} / \mathrm{min})$ at approximately ten-minute intervals were performed, and the micturition threshold volume (MTV) was recorded and used as a dependent variable. After four to five stable recordings, the tibial nerves of both sides were stimulated continuously for five minutes at $10 \mathrm{~Hz}$ and at an intensity of three times the threshold for a-motor axons. Six same stimulations were applied repeatedly, with an interval of five minutes between each stimulation. Mean MTV was calculated on the basis of several cystometries in each half-hour period before, during, and after the six repeated TNS.

Results: During the experiment, all the animals survived in good condition with relatively stable micturition reflexes, and a significant increase in MTV was detected after TNS. The strongest effect (mean $=178 \%$ ) was observed during the first 30 minutes after six repeated stimulations. This obvious threshold increase remained for at least five hours.

Conclusions: A prolonged poststimulation modulatory effect on the micturition reflex was induced by short-term repeated TNS in decorticated rats. This study provides a theoretical explanation for the clinical benefit of TNS in patients with overactive bladder and suggests decorticated rats as a promising model for further investigation of the neurophysiological mechanisms underlying the bladder inhibitory response induced by TNS.
\end{abstract}

Keywords: Cystometry, decorticated rat, micturition reflex, modulatory effects, tibial nerve stimulation Conflict of Interest: The authors reported no conflict of interest.

\section{INTRODUCTION}

Tibial nerve stimulation (TNS), defined as a minimally invasive procedure by posterior percutaneous or transcutaneous tibial nerve stimulation, delivers electrical stimulation to the sacral nerve plexus. Activating this afferent may induce a sympathetic inhibitory pathway and central inhibitory connection to the parasympathetic preganglionic neurons to suppress bladder overactivity. ${ }^{1-3}$ Clinical studies have demonstrated that TNS induces a prolonged poststimulation inhibitory effect on bladder activity, which lasts for days or weeks. ${ }^{4-8}$ Therefore, TNS has been listed as an optional therapy in the American Urological Association treatment guidelines as the third-line treatment for overactive bladder (OAB). ${ }^{9}$

Despite the clinical benefits of this procedure, the underlying mechanisms remain to be clarified owing to significant differences in results obtained from animal and clinical studies. Experimental studies on urinary bladder physiology in cats and rats demonstrated that TNS could induce an immediate bladder inhibitory effect, as indicated by increased bladder capacity and decreased micturition frequency. ${ }^{3,10-12}$ However, these poststimulation effects only last for a short period (approximately 1.5-2 hours) after each stimulation. Moreover, most of the existing studies have used anesthetized animals for micturition reflex evaluation, but the

Address correspondence to: Jianwen Zeng, MD, Department of Urology, the Sixth Affiliated Hospital of Guangzhou Medical University, Qingyuan People's Hospital, Qingyuan 511518, China. Email: zengjwen@gzhmu.edu.cn

1 Department of Urology, The Sixth Affiliated Hospital of Guangzhou Medical University, Qingyuan People's Hospital, Qingyuan, China; and

2 Department of Clinical and Experimental Medicine, Linköping University, Linköping, Sweden

andicates equal contribution.

For more information on author guidelines, an explanation of our peer review process, and conflict of interest informed consent policies, please see the journal's Guide for Authors.

Source(s) of financial support: This study was supported by the Medical Scientific Research Foundation of Guangdong Province, China (grant numbers: 2013A806, B2020011); Natural Science Foundation of Guangdong Province, China (grant number: 2016A030307033); National Natural Science Foundation of China (81802551); and Chinese Postdoctoral Science Foundation (2020M672593). 
accuracy was significantly limited because of the effect of anesthesia on the micturition reflex. Notably, a recent study using an implantable electrical stimulator and a wireless power delivery system showed that continuous TNS at $10 \mathrm{~Hz}$ for 30 minutes induced a poststimulation inhibitory effect of ten hours in unanesthetized conscious rats. ${ }^{13}$ However, conscious animals may be sensitive to other stimuli, such as pain and fear, thus biasing the results. Moreover, it is difficult to keep the stimulator still for a long time in an unanesthetized, vigorous animal for outside bladder stimulation and the body movement of experimental animals may affect the results, leading to a significant between-subject discrepancy. In the study by Park et al, $^{13}$ the poststimulation inhibitory effect showed a large discrepancy in inhibitory duration for each animal (3-25 hours). Therefore, further studies with a more reliable animal model are needed to determine the prolonged poststimulation inhibitory effect of TNS.

We have previously reported that repeated short-term anogenital afferent stimulation in decorticated rats induced a prolonged poststimulation inhibition effect on the micturition reflex for more than five hours, which is well beyond the stimulation period, suggesting decorticated rats as a promising model for studying TNS effect on the micturition reflex. ${ }^{14}$ Inspired by this positive finding, this experimental study was designed to determine whether shortterm repeated TNS induces a prolonged modulation effect on the micturition reflex in decorticated rats. These findings provide experimental evidence to support the clinical application of TNS in the treatment of lower urinary tract dysfunction, especially $O A B$.

\section{MATERIALS AND METHODS}

\section{Animals and Surgical Procedures}

The experiment was carried out on 15 adult female rats (SpragueDawley, 250-350 g). Under anesthesia with methohexitone (Brietal, $70 \mathrm{mg} / \mathrm{kg}$ intraperitoneally), the femoral vein and artery were cannulated to allow fluid injections and blood pressure recordings, and a tracheotomy was performed for artificial respiration. Then, the animals were decorticated following the reported procedures. ${ }^{15}$ In brief, after craniectomy and dura excision, the full decortication was performed by gentle suction, using a standard drawn glass pipette connected to a vacuum pump, and most of the diencephalon and the entire hippocampus were spared. The fenestra was then filled with hemostatic cotton or gelatin sponge soaked with thrombin to minimize bleeding during the experiment. This procedure rendered the animals unconscious without need for further anesthesia.

For recordings, the animals were paralyzed by a continuous intravenous infusion of pancuronium bromide $(0.3 \mathrm{mg} / \mathrm{kg} . \mathrm{h})$ and artificially ventilated with end-expiratory $\mathrm{CO}_{2}$ adjusted to approximately $3.5 \%$. Care was taken to ascertain that the paralyzed animals were adequately unconscious by regularly controlling that strong paw-pinches failed to induce changes in blood pressure and heart rate. Their body temperature was maintained at approximately $38{ }^{\circ} \mathrm{C}$ by a feedback-controlled heating lamp.

These animals were killed at the end of the experiment by an overdose of anesthetics followed by severance of the heart. All the surgical and experimental procedures were approved by the animal research ethics committee of the Sixth Affiliated Hospital of Guangzhou Medical University.

\section{Cystometry and Tibial Nerve Stimulation}

The experimental arrangement is shown in Figure 1a. Bilateral tibial nerve stimulation was performed by percutaneously inserting two pairs of needle electrodes close to the tibial nerves at the level of the medial malleolus from cathode to anode (1 cm apart). A polyethylene catheter ( $8 \mathrm{~F})$ with a side hole close to the tip was inserted into the bladder through a slit in the proximal urethra and fixed by a 3-0 silk ligature around the catheterized urethra. The catheter was connected to an infusion pump and a pressure transducer for cystometry recordings.

Constant flow cystometries were performed repeatedly with body-warm saline $(0.07 \mathrm{~mL} / \mathrm{min})$ at about ten-minute intervals. As soon as the bladder contracted, the infusion was turned off, and the catheter was opened. Care was taken to empty the bladder after each cystometry by lowering the catheter outlet below the level of the bladder. The micturition threshold volume (MTV) was defined as the amount of fluid infused into the bladder at the onset of micturition contraction and was used as a dependent variable. For control, four to five cystometry recordings were performed before TNS. Then, TNS was applied immediately after the cystometrogram (CMG) with the catheter open, and the stimulation was continuously performed for five minutes at a frequency of 10 $\mathrm{Hz}$ and at an intensity of three times the threshold for a-motor axons determined by the induced obvious toe movement. These electrical stimulation parameters were chosen on the basis of our previous study and another study. ${ }^{14,16}$ The same stimulation procedures were repeated six times with five-minute intervals, and one cytometry was performed between each stimulation. The bladder was emptied with an open catheter during the stimulation. The modulatory effect of TNS was defined as the mean of MTV calculated from several cystometries in each half-hour period before, during, and after the TNS.

\section{Statistical Analysis}

For group comparison, data were normalized and expressed as a percentage of the control using the Wilcoxon signed-rank test. Data from different animals (mean \pm SE) were compared by repeated measures one-way analysis of variance. A $p$ value of $\leq 0.05$ was considered statistically significant. Statistical analyses were performed with SPSS version 19.0 (IBM Corp, Armonk, NY).

\section{RESULTS}

All decorticated rats survived in a good state for one to three days with relatively stable micturition reflexes. The stability of bladder contractility was ascertained by four to five sessions of control cystometries, as indicated by $<10 \%$ difference in MTV between each cystometry. TNS induced a remarkable inhibitory effect on the micturition reflex, as manifested by a significant increase in MTV. Typical cystometry recordings were detected before and after TNS (Fig. 2). A minimal increase in bladder pressure during the infusion (until a micturition contraction) was observed at a bladder volume of $0.31 \mathrm{~mL}$ (Fig. 2a) and $0.44 \mathrm{~mL}$ (Fig. 2b). Record A was obtained ten minutes before TNS, and record $B$ was obtained five minutes immediately after TNS. The stimulation induced a $142 \%$ increase in MTV compared with that in the control.

The time course of changes in MTV induced by TNS is illustrated in Figure 3. Five cystometries were performed to ascertain stable bladder contraction before the stimulation. In this representative case, the MTV was between 0.1 and $0.15 \mathrm{~mL}$, which was a reasonable control for the following test experiments. The inhibitory effect of TNS was demonstrated immediately after the first 
a

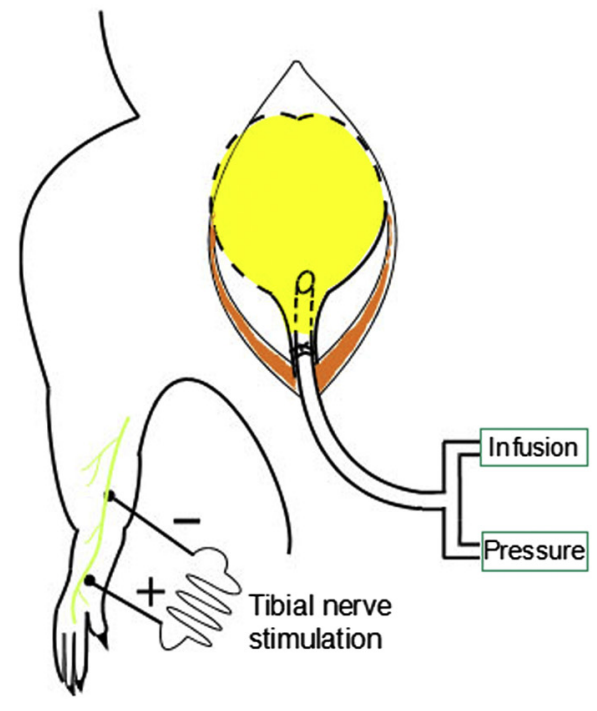

b

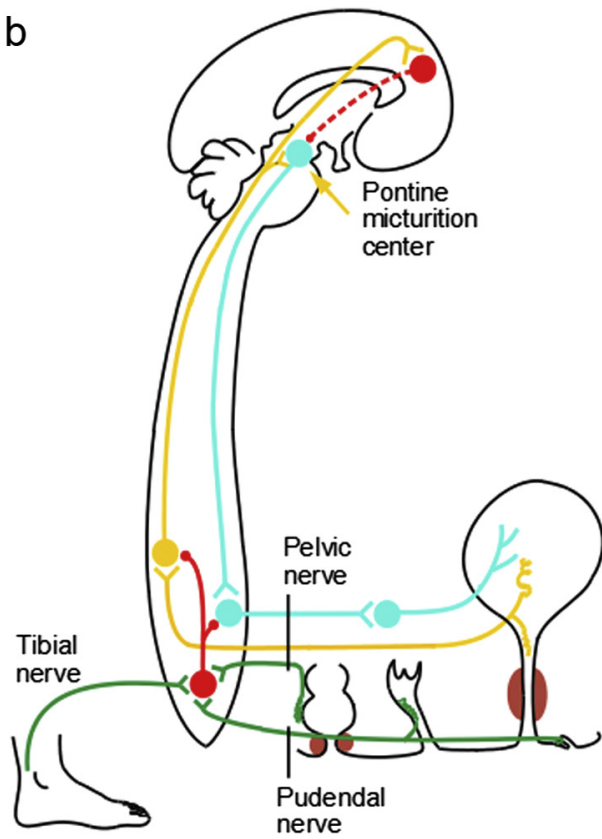

Figure 1. Schematic diagram of (a) experimental arrangements in rats and (b) inhibitory control systems of the bladder in humans. The afferents from the tibial nerve, anal canal, and vaginal mucosae not only affect the motor output from the spinal cord but also send the signals up to the pontine center and the cerebral cortex. All these pathways prevent unintended micturition. Yellow indicates bladder afferents, blue indicates bladder efferent, green indicates inhibitory afferents from the indicated organs, and red indicates inhibitory interneurons. For better presentation, (a) only one side of the stimulation diagram is shown, and the stimulation diagram on the other side is the same. During the stimulation, the bladder was empty with the catheter open. [Color figure can be viewed at www.neuromodulationjournal.org]

five-minute stimulation (eg, the MTV increased from 0.15 to 0.27 $\mathrm{mL}$ ). This increasing tendency persisted in the next four repeated stimulations, and the MTV peaked at $0.38 \mathrm{~mL}$ after five stimulations. After six stimulations, a significant increase in MTV persisted for more than five hours.

A prolonged poststimulation inhibitory effect of TNS on the micturition reflex was demonstrated in another representative series of CMG recordings (Fig. 4). In this case, there were no significant changes in MTV in the first four consecutive recordings compared with the baseline (before the stimulation) (mean: $0.39 \pm 0.04 \mathrm{~mL}$ ), whereas a significant increase in MTV was observed immediately after each five-minute stimulation, and repeated TNS (six times, half hour/each time) induced a prolonged poststimulation inhibitory effect for more than five hours. The normalized values correspond to the pooled data histogram (Fig. 5).

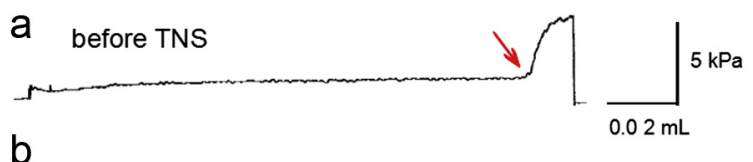

after TNS

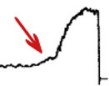

Figure 2. CMGs were recorded (a) before and (b) after TNS. The bladder was filled at a constant rate $(0.07 \mathrm{~mL} / \mathrm{min})$ with body-warm saline. Record A was obtained ten minutes before TNS, and record B was obtained five minutes immediately after TNS. TNS was delivered for five minutes at $10 \mathrm{~Hz}$ and at an intensity of three times the threshold for a-motor axons (same as in other figures). Note that increased MTV was induced by TNS after stimulation (0.44 vs $0.31 \mathrm{~mL}, 142 \%$ ). Red arrows point to MTV. [Color figure can be viewed at www.neuromodulationjournal.org]
For better comparison, data of all animals ( 675 cystometries of 15 rats) were pooled (Fig. 5). To reduce scatter due to large variations in MTV among the animals, the values were normalized before pooling and expressed as a percentage of the mean control value of each animal. TNS induced a significant increase in MTV for all animals during and after TNS $(p<0.01)$. The MTV increased to $160 \%$ of the control value after the first stimulation and continued to increase to $180 \%$ of the control value after the second stimulation. After six repeated stimulations, the mean MTV reached a peak of $190 \%$ of the control value at 30 minutes and remained at about $150 \%$ of the control value for three hours after the stimulation. The powerful inhibitory effect of TNS on the micturition reflex persisted for five hours (Fig. 5).

\section{DISCUSSION}

This study has demonstrated that repeated short-period TNS induced a prolonged poststimulation inhibition effect on the micturition reflex for five hours in unanesthetized, decorticated rats. The decorticated rat model avoided the confounding effects of anesthesia and was proved to be a reliable animal model for studying the effects of nerve stimulation. Altogether, our study provides reliable experimental evidence to support the clinically beneficial effects of repeated short-period TNS in the treatment of lower urinary tract dysfunction.

In this study, we showed that TNS for five minutes at a frequency of $10 \mathrm{~Hz}$ and at an intensity of three times the threshold for amotor axons induced a $160 \%$ increase in MTV compared with the control, immediately after the first TNS. The prolonged poststimulation inhibitory effect was observed after six repeated 


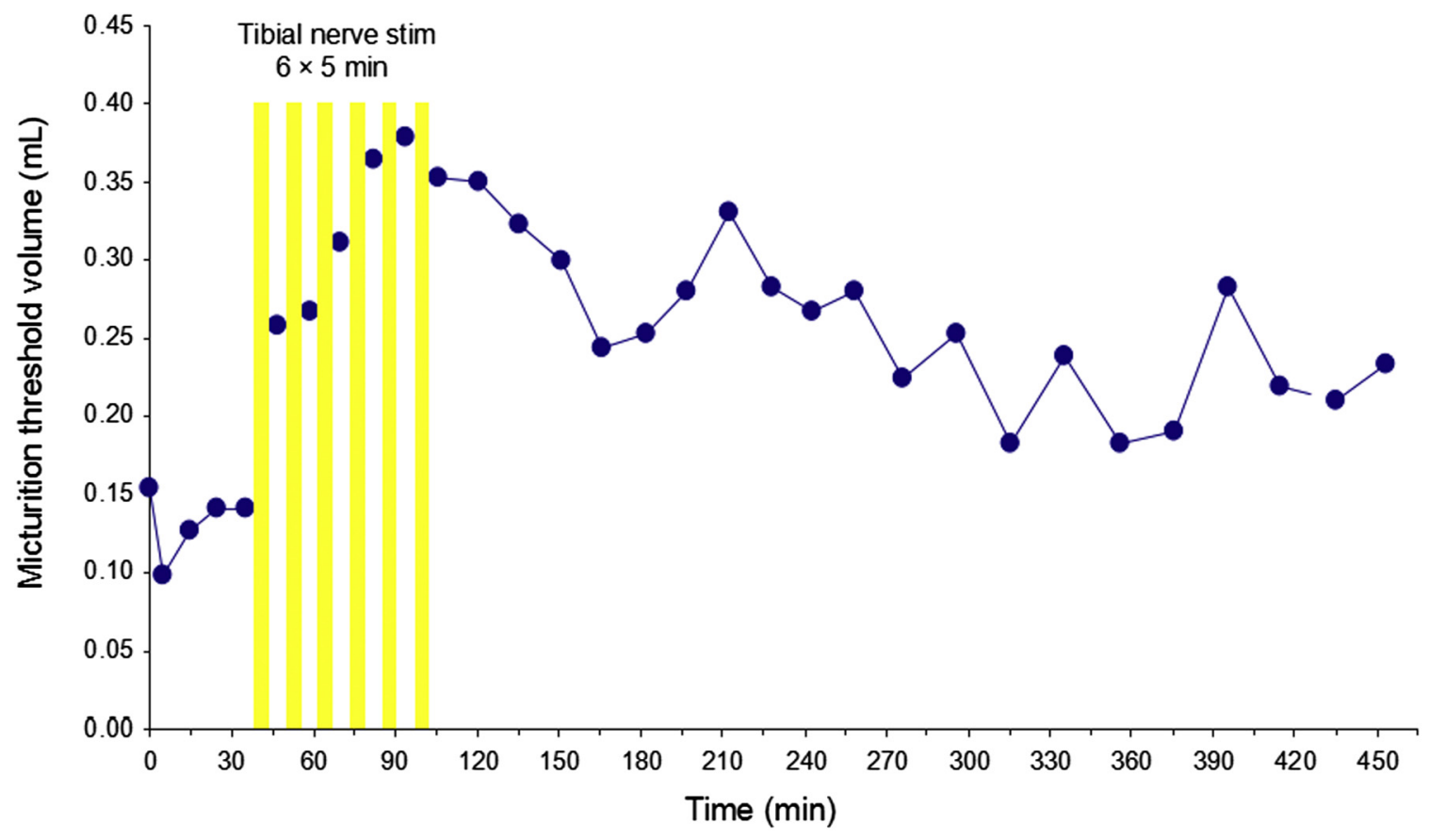

Figure 3. Changes in MTV after TNS. Each point in the plot represents the MTV of each cystometry at the indicated time interval. Each bar indicates a five-minute stimulation. [Color figure can be viewed at www.neuromodulationjournal.org]

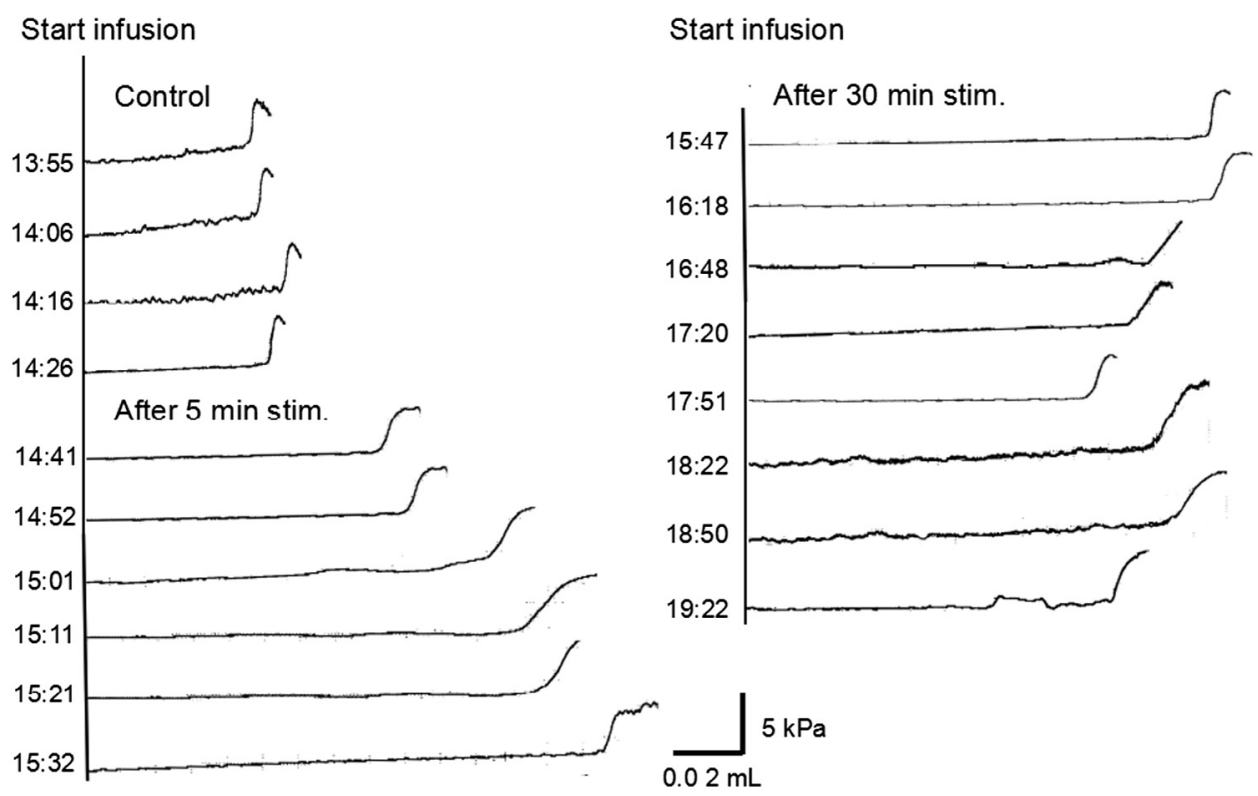

Figure 4. A series of representative CMG recordings in another animal (different from Fig. 3) showed a prolonged poststimulation inhibitory effect of TNS on the micturition reflex. MTV did not significantly change in the first four consecutive CMGs compared with the baseline (before the stimulation) (mean: $0.39 \pm 0.04 \mathrm{~mL}$ ). There was a significant increase in MTV after each five-minute stimulation (indicated by "After 5 min stim.") and after six TNS (five minutes on and five minutes off for six times, indicated by "After 30 min stim."). For simplification, CMGs after repeated stimulations were omitted following every two consecutive CMGs. The normalized values correspond to pooling data plotted in Figure 5. Infusion rate was $0.07 \mathrm{~mL} / \mathrm{min}$. Bladder pressure and time calibration in the lower right corner refer to all CMG traces. stim., stimulation.

stimulations, with a significant increase in MTV for more than five hours, longer than that reported in other studies with similar stimulation parameters. ${ }^{10,11,17}$ For example, previous studies reported that TNS induced a poststimulation inhibition effect on the micturition reflex lasting 50 minutes to one hour in rats. ${ }^{11,17}$
Tai et al $^{10}$ reported a poststimulation inhibitory effect on bladder activity that lasted for more than two hours after 30 minutes of TNS at 5 to $30 \mathrm{~Hz}$ in cats. Although the observation time was different between the studies, it was unlikely to have contributed to the differences in inhibitory durations between the studies 


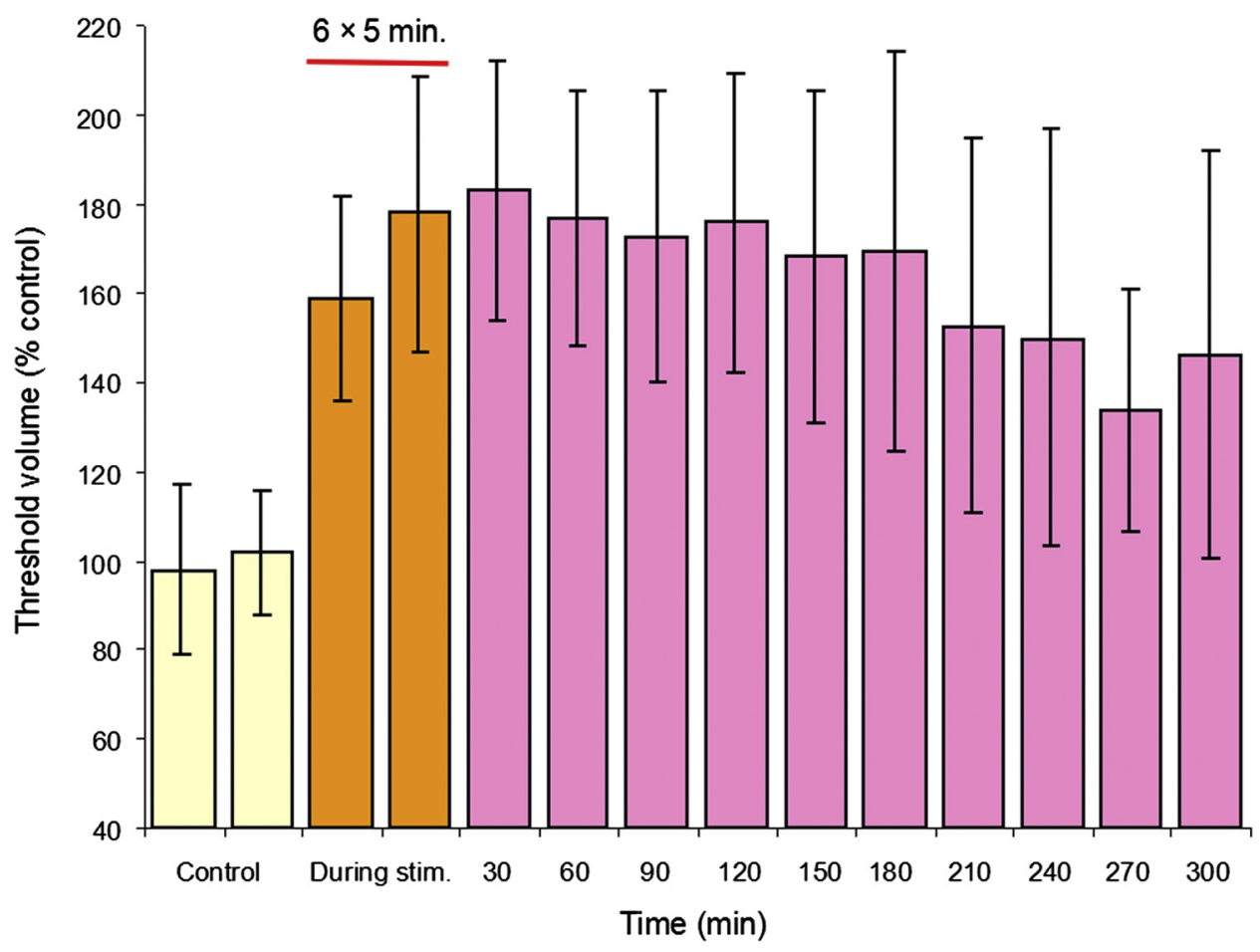

Figure 5. Time course of tibial nerve stimulation-induced changes in MTV in all the animals. MTV measurement in each rat (three CMG values) was determined every 30 minutes. For better comparison, data of all animals ( 675 cystometries of 15 rats) were pooled and expressed as a percentage of the mean control value. Each bar represents a normalized threshold value $( \pm$ SEM) in each half-hour period before, during, and after the stimulations. Six repeated TNS with five-minute intervals were delivered, as indicated by a horizontal red $\operatorname{bar}(N=15 ; p<0.01$ in all cases). stim., stimulation. [Color figure can be viewed at www.neuromodulationjournal.org]

because the observation usually continued until the cessation of the effect in each experiment. More probably, these differences might have been due to the use of anesthesia in the mentioned studies because anesthesia might attenuate the neurophysiological response to TNS. Moreover, the stimulation modality may have also contributed to the differences in inhibitory duration between the studies, although the parameters used were roughly similar. In this study, we used a repeated short-period stimulation (ie, a five minutes on and five minutes off stimulation pattern) rather than continuous stimulation because such an algorithm is effective for other central synapses. ${ }^{18}$ Besides, a break between each stimulation period is necessary for the bladder to recover from the previous contraction. From a clinical perspective, a proper interval between each stimulation session is important to activate the inhibitory effect on the micturition reflex, and it may also allow patients to better adapt to the electrical stimulation and improve treatment compliance. ${ }^{19}$ In this context, this repeated stimulus modality has been applied in clinical practice, and the beneficial effects have been observed in patients with $O A B$, although the stimulus sessions and recovery intervals (ie, 30 minutes each day or week) were longer than those in rats. ${ }^{20-22}$ Taken together, these findings demonstrate a prolonged inhibitory effect of short-term repeated TNS on the micturition reflex and confirm short-term repeated TNS as a promising therapeutic strategy for lower urinary tract dysfunction.

It is well-known that the micturition reflex is highly sensitive to anesthesia, which will bias the results, especially when long-term poststimulation effects are studied. ${ }^{23}$ Therefore, the accuracy of most existing studies is limited because of the use of anesthetized animals for micturition reflex evaluation. ${ }^{17}$ Although Park et $\mathrm{al}^{13}$ have reported a poststimulation inhibitory effect of TNS for an average of ten hours in unanesthetized active rats by using an implantable electrical stimulator and a wireless power delivery system, it is difficult to rule out interference from other stimuli in conscious animals, such as body movement, pain, and fear, resulting in significant between-subject discrepancy and inaccurate results. In this context, the decorticated rat model can overcome these limitations. It renders the animal unconscious and facilitates survival in a good state for one to three days with relatively stable micturition reflexes. This model has been proved to be reliable and effective for the study of poststimulation effects of anogenital afferent stimulation on the micturition reflex in our previous study. ${ }^{14}$ Accordingly, the current study using decorticated rats also obtained stable CMGs before the stimulation and observed a longlasting increase in MTV after TNS, with low between-animal discrepancy. Taken together, our findings provided convincing evidence that short-term repeated TNS induced long-term modulatory effects on the micturition reflex and suggested decorticated rats as a promising model for studying the neurophysiological mechanisms underlying the bladder inhibitory response to TNS.

Neuromodulation therapies use electrical stimulation to activate specific nerves and can modulate bladder activity in the treatment of $O A B$ and other lower urinary tract disorders. TNS is one of such neuromodulation procedures because of its inhibitory effect on micturition reflexes. Different TNS protocols exist, including direct and indirect approaches. Direct TNS approaches include implanted stimulation devices over the tibial nerve in humans ${ }^{24}$ and bipolar or tripolar cuff electrodes placed around the nerve in rats. ${ }^{25}$ Indirect TNS approaches include implanting wireless driver microstimulators, ${ }^{26}$ percutaneous TNS, or transcutaneous tibial nerve 
stimulation. ${ }^{4-6}$ The TNS technique applied in this study is similar to that used in the clinic (ie, inserting two needles cranially $3-4 \mathrm{~cm}$ to the medial tibial malleolus and stimulating by connecting the electrostimulator). We chose this technique on the basis of an idea from the Chinese acupuncture treatment guidelines, which recommend needle insertion into the tibial nerve corresponding to the Sanyinjiao point (Fig. 1a), and the nerve dissection was performed before the experiment (Fig. 1). Despite a questionable difference in TNS effects between surface-electrode and nerve-cuff approaches as reported by Kovacevic et al, ${ }^{21}$ all the procedures have similar inhibitory effects on the micturition reflex when suitable stimulation parameters are applied. The so-called advanced techniques of direct nerve stimulation did not show more beneficial effects than indirect nerve stimulation techniques. In this study, the significant increase in MTV suggests that our TNS procedure in rats is applicable.

Tibial afferent nerves project to the segments of the lumbosacral spinal cord and receive inputs from bladder afferents traveling in the pelvic nerves. ${ }^{3}$ Activating these systems by electrical stimulation produces central inhibition of the micturition reflex, as demonstrated in this study; the effect is very similar to that of anogenital afferent stimulation. This inhibition occurs at least at the spinal level. In addition, all these afferent systems not only affect the motor output from the spinal cord but also send the signals up to the pontine center and cerebral cortex. Notably, all these pathways are well-defined to be involved in the prevention of unintended micturition (Fig. 1b). ${ }^{1,27}$

The stimulation intensity at three times the threshold for a-motor axons (the threshold intensity for inducing a toe movement) was applied in this study because this TNS intensity is sufficient for activating both $A \delta$ and C-fiber afferents, as demonstrated before the experiment. Other studies also revealed that this current had an inhibition effect on isovolumetric bladder contractions in cats ${ }^{3,27}$ and a poststimulation inhibition effect indicated by increased bladder capacity and compliance in rats. ${ }^{11}$ We hypothesize that this somato-vesical inhibition effect is involved in both supraspinal and spinal circuits of the bladder via $A \delta$ and $C$-fiber micturition reflex pathways, respectively.

Such a prolonged (five-hour) poststimulation modulation effect on the micturition reflex would be better explained by the modulation of the synaptic transmission in the central micturition reflex pathway. This idea is supported by our previous study, which showed that intravesical electrical stimulation-induced enhancement of the micturition reflex could be prevented by systemic administration of a specific and competitive $N$-methyl-D-aspartate (NMDA) antagonist, CPPene. ${ }^{28}$ Activation of NMDA receptors has been demonstrated to play a role in the modulation of synaptic efficacy. The mechanism of long-term potentiation is associated with the enhancement of excitatory synaptic transmission in the central nervous system. Thus, this bladder inhibitory effect of TNS might be due to a negative modulation of excitatory synapses in the central micturition reflex pathway. Notably, a prolonged decrease in synaptic efficacy of excitatory synapses, referred to as long-term depression (LTD), has been observed in the hippocampus after intense activation of an inhibitory input to target cells. ${ }^{28,29}$ In addition, other central processes, such as opioid receptor-associated processes in the brainstem, have also been reported to be involved in TNS-induced inhibition of bladder overactivity in cats. ${ }^{30}$ Given that the effects were observed in animals lacking cortical control of the micturition reflex, higher cognitive functions were demonstrated to not be involved in the bladder inhibitory effect of TNS. Thus, the effects observed in some patients with $O A B$ following repeated sessions of TNS may, at least in part, result from LTD-like modulations of synaptic transmission in the subcortical micturition reflex pathway. ${ }^{29,31}$

The major limitation of the current study is the lack of continuous stimulation controls. Although short-term repeated TNS showed a promising inhibitory effect, it remains unclear whether it is better than continuous stimulation because of the lack of study controls. Therefore, further studies with continuous stimulation controls are warranted to compare the effect of short-term repeated TNS with that of conventional continuous stimulation and to investigate the underlying mechanisms contributing to the differences.

\section{CONCLUSIONS}

A prolonged increase in MTV was demonstrated by repeated short-period TNS in decorticated rats. This inhibitory poststimulation modulation effect on the micturition reflex might result from the activation of inhibitory neural mechanisms in the central micturition reflex pathway. Our findings provide a theoretical explanation for the clinically beneficial effects of TNS in patients with $O A B$ and suggest decorticated rats as a promising model for further investigation of the neurophysiological mechanisms underlying the bladder inhibitory response induced by TNS.

\section{Authorship Statements}

Junyan Mai, Junhao Liao, Yuying Zhang, and Jianwen Zeng conceived the study and its design and assisted with the manuscript preparation, data collection, and editing. Yuying Zhang and Baoyi Zhu contributed to data analysis and reviewed the manuscript. Chonghe Jiang and Sivert Lindström contributed to the discussion and reviewed the manuscript. All the authors read and approved the final manuscript.

\section{How to Cite This Article}

Mai J., Liao J., Zhang Y., Zhu B., Jiang C., Lindström S., Zeng J. 2022. Prolonged Inhibitory Effects of Repeated Tibial Nerve Stimulation on the Micturition Reflex in Decorticated Rats.

Neuromodulation 2022; 25: 1115-1121.

\section{REFERENCES}

1. Shefchyk SJ. Sacral spinal interneurones and the control of urinary bladder and urethral striated sphincter muscle function. J Physiol. 2001;533:57-63.

2. Jiang $\mathrm{CH}$, Mazières L, Lindström S. Gating of the micturition reflex by tonic activation of bladder cold receptors in the cat. Neurourol Urodyn. 2009;28:555-560.

3. Tai C, Chen M, Shen B, Wang J, Roppolo JR, de Groat WC. Irritation induced bladder overactivity is suppressed by tibial nerve stimulation in cats. J Urol. 2011;186:326-330.

4. Gaziev G, Topazio L, lacovelli V, et al. Percutaneous tibial nerve stimulation (PTNS) efficacy in the treatment of lower urinary tract dysfunctions: a systematic review. BMC Urol. 2013;13:61.

5. Peters KM, Carrico DJ, Wooldridge LS, Miller CJ, MacDiarmid SA. Percutaneous tibial nerve stimulation for the long-term treatment of overactive bladder: 3-year results of the STEP study. J Urol. 2013;189:2194-2201.

6. Del Río-Gonzalez S, Aragon IM, Castillo E, et al. Percutaneous tibial nerve stimulation therapy for overactive bladder syndrome: clinical effectiveness, urodynamic, and durability evaluation. Urology. 2017:108:52-58.

7. Patidar N, Mittal V, Kumar M, Sureka SK, Arora S, Ansari MS. Transcutaneous posterior tibial nerve stimulation in pediatric overactive bladder: a preliminary report. J Pediatr Urol. 2015;11:351.e1-351.e6.

8. Tudor KI, Seth $\mathrm{JH}$, Liechti MD, et al. Outcomes following percutaneous tibial nerve stimulation (PTNS) treatment for neurogenic and idiopathic overactive bladder Clin Auton Res. 2020;30:61-67. 
9. Lightner DJ, Gomelsky A, Souter L, Vasavada SP. Diagnosis and treatment of overactive bladder (non-neurogenic) in adults: AUA/SUFU guideline Amendment 2019. J Urol. 2019;202:558-563.

10. Tai C, Shen B, Chen M, Wang J, Roppolo JR, de Groat WC. Prolonged poststimulation inhibition of bladder activity induced by tibial nerve stimulation in cats. Am J Physiol Renal Physiol. 2011;300:F385-F392.

11. Matsuta Y, Roppolo JR, de Groat WC, Tai C. Poststimulation inhibition of the micturition reflex induced by tibial nerve stimulation in rats. Physiol Rep. 2014;2:e00205.

12. Li S, Theisen K, Browning J, et al. Bladder underactivity after prolonged stimulation of somatic afferent axons in the tibial nerve in cats. Neurourol Urodyn. 2018;37:2121-2127.

13. Park $E$, Lee JW, Kim T, et al. The long-lasting post-stimulation inhibitory effects of bladder activity induced by posterior tibial nerve stimulation in unanesthetized rats. Sci Rep. 2020;10:19897.

14. Chen H, Zeng J, Zeng P, Jiang C, Xie K, Lindström S. Repeat periods of electrica stimulation prolong the modulation of the micturition reflex in the rat. Neurourol Urodyn. 2018;37:2480-2486.

15. Wilson JR, Vardaris RM, Schweikert GE 3rd. Technique for chronic electrode or cannula implantation in decorticate animals. Physiol Behav. 1975;14:875-877.

16. Choudhary $M$, van Mastrigt $R$, van Asselt E. Effect of tibial nerve stimulation on bladder afferent nerve activity in a rat detrusor overactivity model. Int J Urol. 2016;23:253-258.

17. Paquette JP, Yoo PB. Recruitment of unmyelinated C-fibers mediates the bladderinhibitory effects of tibial nerve stimulation in a continuous-fill anesthetized rat model. Am J Physiol Renal Physiol. 2019;317:F163-F171.

18. Luber B, Lisanby SH. Enhancement of human cognitive performance using transcranial magnetic stimulation (TMS). Neuroimage. 2014;85:961-970.

19. Chen G, Larson JA, Ogagan PD, et al. Post-stimulation inhibitory effect on reflex bladder activity induced by activation of somatic afferent nerves in the foot. $J$ Urol. 2012;187:338-343.
20. Capitanucci ML, Camanni D, Demelas F, Mosiello G, Zaccara A, De Gennaro M Long-term efficacy of percutaneous tibial nerve stimulation for different types of lower urinary tract dysfunction in children. J Urol. 2009;182(suppl 4):2056-2061.

21. Kovacevic M, Lin R, Yoo PB. An enhanced method of transcutaneously stimulating the tibial nerve for the treatment of overactive bladder. Ann Biomed Eng. 2017;45:2605-2613.

22. Ramírez-García I, Blanco-Ratto L, Kauffmann S, Carralero-Martínez A, Sánchez E. Efficacy of transcutaneous stimulation of the posterior tibial nerve compared to percutaneous stimulation in idiopathic overactive bladder syndrome: randomized control trial. Neurourol Urodyn. 2019;38:261-268.

23. Rudy DC, Downie JW, MCAndrew JD. alpha-Chloralose alters autonomic reflex function of the lower urinary tract. Am J Physiol. 1991;261:R1560-R1567.

24. MacDiarmid S, Staskin DR, Lucente $V$, et al. Feasibility of a fully implanted, nicke sized and shaped tibial nerve stimulator for the treatment of overactive bladder syndrome with urgency urinary incontinence. J Urol. 2019;201:967-972.

25. Choudhary M, van Mastrigt R, van Asselt E. Inhibitory effects of tibial nerve stim ulation on bladder neurophysiology in rats. Springerplus. 2016;5:35.

26. Moazzam Z, Duke AR, Yoo PB. Inhibition and excitation of bladder function by tibial nerve stimulation using a wirelessly powered implant: an acute study in anesthetized cats. J Urol. 2016;196:926-933.

27. de Groat WC. A neurologic basis for the overactive bladder. Urology. 1997;50:36-52 [discussion: 53-36].

28. Jiang $\mathrm{CH}$. Modulation of the micturition reflex pathway by intravesical electrical stimulation: an experimental study in the rat. Neurourol Urodyn. 1998;17:543-553.

29. Lüscher C, Malenka RC. NMDA receptor-dependent long-term potentiation and long-term depression (LTP/LTD). Cold Spring Harb Perspect Biol. 2012;4:a005710.

30. Ferroni MC, Slater RC, Shen $B$, et al. Role of the brain stem in tibial inhibition of the micturition reflex in cats. Am J Physiol Renal Physiol. 2015;309:F242-F250.

31. Bear MF, Malenka RC. Synaptic plasticity: LTP and LTD. Curr Opin Neurobiol. 1994:4:389-399. 